\title{
Subsurface Imaging of Grain Microstructure Using Picosecond Ultrasonics
}

\author{
M. Khafizov ${ }^{1}$, J. Pakarinen ${ }^{2}$, L. He ${ }^{3}$, H. B. Henderson ${ }^{4}$, M. V. Manuel ${ }^{4}$, A. T. Nelson ${ }^{5}$, B. J. \\ Jaques $^{6}$, D. P. Butt ${ }^{6,7}$, D. H. Hurley ${ }^{3}$ \\ ${ }^{1}$ Mechanical \& Aerospace Engineering, The Ohio State University, Columbus, Ohio 43210, USA \\ ${ }^{2}$ Belgian Nuclear Research Center (SCK-CEN), Boeretang 200, B-2400 Mol, Belgium \\ ${ }^{3}$ Idaho National Laboratory, Idaho Falls, Idaho 43215, USA \\ ${ }^{4}$ Materials Science \& Engineering, University of Florida, Gainesville, Florida 32611, USA \\ ${ }^{5}$ Los Alamos National Laboratory, Los Alamos, New Mexico 87545, USA \\ ${ }^{6}$ Materials Science \& Engineering, Boise State University, Boise, Idaho 83725, USA \\ ${ }^{7}$ Center for Advanced Energy Studies, Idaho Falls, Idaho 83401, USA
}

\begin{abstract}
We report on imaging subsurface grain microstructure using picosecond ultrasonics. This approach relies on elastic anisotropy of crystalline materials where ultrasonic velocity depends on propagation direction relative to the crystal axes. Picosecond duration ultrasonic pulses are generated and detected using ultrashort light pulses. In materials that are transparent or semitransparent to the probe wavelength, the probe monitors gigahertz frequency Brillouin oscillations. The frequency of these oscillations is related to the ultrasonic velocity and the optical index of refraction. Ultrasonic waves propagating across a grain boundary experience a change in velocity due to a change in crystallographic orientation relative to the ultrasonic propagation direction. This change in velocity is manifested as a change in the Brillouin oscillation frequency. Using the ultrasonic propagation velocity, the depth of the interface can be determined from the location in time of the transition in oscillation frequency. A subsurface image of the grain boundary is obtained by scanning the beam along the surface. We demonstrate this subsurface imaging capability using a polycrystalline $\mathrm{UO}_{2}$ sample. Cross section liftout analysis of the grain boundary using electron microscopy was used to verify our imaging results.
\end{abstract}

Keywords: picosecond ultrasonics, grain orientation, boundary characterization * Corresponding authors Marat Khafizov, Khafizov.1@osu.edu and David Hurley, david.hurley@inl.gov 


\section{Introduction}

Grain boundaries serve an important role in defining the physical properties of ceramic materials used in the energy industry.(1) For example, ion transport in solid oxide fuels is affected by atomic segregation at grain boundaries.(2) Grain boundaries can also significantly impact thermal transport in materials used for thermal management as well as high burnup nuclear fuels.(3-6) In addition mechanical properties of energy materials are often influenced by the diffusion of atoms in high stress and high temperature environments.(7, 8) Grain boundaries, under these extreme conditions can act as barriers or provide a pathway for atomic diffusion.( 9 , 10) Many of the above mentioned phenomena are strongly influenced by the local environment around grain boundaries.(11)

A central complexity in understanding these fundamental mechanisms is that grain boundary character evolves with time. In this regard, grain boundaries attract impurities and other defects that have further implications on determining material behavior.(12) Additionally the boundaries themselves undergo motion under external stimuli such as mechanical deformation, radiation and high temperatures. $(7,13)$ Grain boundary motion and the associated change in crystal misorientation across the boundary can significantly influence transport and mechanical properties. $(14,15)$ To better understand the evolution of boundary structure under external stimuli, there is a need for new experimental techniques that can be used to investigate them nondestructively.(16)

Currently the majority of grain boundary evolution studies have been limited to imaging the boundary line on the surface. $(17,18)$ For these investigations, typically a bicrystal is subjected to deformation and the motion of the boundary on the free surface is visualized using scanning electron microscopy (SEM). This observation mode can be influenced by surface effects and 
may not accurately represent the behavior of the boundary in the bulk. Thus availability of techniques providing three-dimensional (3D) structural information is important for understanding bulk behavior. Methods based on advanced X-ray synchrotron sources are able to provide volumetric grain microstructure.(19),(20) However, limited availability of synchrotron sources prevents wide spread application of X-ray approaches. A focused ion beam instrument combined with electron backscattering diffraction can be used to obtain a 3D image of the grain boundary structure. This approach involves reconstruction of the $3 \mathrm{D}$ structure from a series of 2D crystallographic orientation images obtained after repetitive removal of thin layers using focused ion beam milling.(21-23) Destructive serial sectioning makes this approach not suitable for in-situ measurements. Recently it has been shown that grain inclination with respect to the free surface can be extracted from analysis of the diffraction pattern obtained from the area above the grain boundary. However, the depth resolution of this approach is limited to the penetration depth of electrons.(24)

Here we report on an alternative approach, based on picosecond ultrasonics, which is used to visualize depth resolved grain microstructure. This approach has been used previously for depth profiling of elastic inhomogeneities in transparent materials. $(25,26)$ The emphasis here is on mapping the location of a grain boundary below the surface by monitoring changes in ultrasonic velocity caused by a change in crystallographic orientation. We demonstrate this approach using uranium dioxide $\left(\mathrm{UO}_{2}\right)$, an important energy material broadly used in the nuclear energy industry as well as having promising applications in the solar energy industry.(27, 28)

\section{Picosecond ultrasonics and Brillouin oscillations}

For our application picosecond duration ultrasonic pulses propagating normal to the surface are generated by irradiating $\mathrm{UO}_{2}$ sample with an ultrashort laser pulse (pump) having above bandgap 
photon energy. Both thermal expansion and deformation potential mechanisms are responsible for ultrasonic generation. Strong optical absorption ensures generation of short duration ultrasonic pulses.(29) A time delayed probe pulse tuned to a photon energy that is below the bandgap is used to detect the ultrasonic pulses as they propagate into the sample.(30) In materials that are transparent or semitransparent to the probe wavelength, the probe monitors $\mathrm{GHz}$ Brillouin oscillations. The origin of these oscillations comes from interference between two components of the reflected probe pulse as illustrated in Fig. 1. The first is from the reflection off the free surface of the sample and the second is due to photoelastic coupling with the propagating ultrasonic wave. The frequency of the oscillations, $f$, depends on the probe wavelength $(\lambda)$, the index of refraction at the probe wavelength $(n)$ and the ultrasonic velocity in a direction normal to the surface $(v):(30)$

$$
f=\frac{2 n v}{\lambda}
$$

Detection sensitivity is based on the photoelastic effect, where the strain associated with the acoustic wave modifies dielectric properties. It is known that photoelastic coupling is especially pronounced when the energy of the probe is comparable to the semiconducting gap of the material. The bandgap of $\mathrm{UO}_{2}$ is $\sim 2.2 \mathrm{eV}$. Accordingly we tune our probe and pump photon energies to be $1.5 \mathrm{eV}(800 \mathrm{~nm})$ and $3 \mathrm{eV}(400 \mathrm{~nm})$ respectively For this combination, the pump is strongly absorbed by the sample and the probe beam is able to penetrate into the sample several tens of microns. A similar approach has been extensively used to observe Brillouin oscillations in GaAs and was implemented to characterize defects in ion irradiated GaAs.(31)

The emphasis here is to utilize elastic anisotropy to image subsurface grain microstructure. $\mathrm{UO}_{2}$ exhibits moderate elastic anisotropy which makes it a good system for demonstration of volumetric imaging of grain boundary structure. In an elastically anisotropic material, the 
velocity of ultrasonic wave depends on the direction of propagation with respect to its crystallographic axes. As a result, the frequency of Brillouin oscillations depends on the grain orientation. The maximum depth resolution of this approach, using our simple 1D analysis, is related to acoustic diffraction and ultimately limited by optical absorption of the probe beam. In the very near field of the acoustic source, acoustic diffraction can safely be neglected. This region is defined by the lateral dimensions of the source and for the case considered here (spot size $\sim 2 \mu \mathrm{m}$ ) the maximum probe depth is approximately 10 microns.

\section{Calculation of ultrasonic velocity in single crystals}

Propagation of an ultrasonic wave is described by the elastic wave equation:

$$
\rho \frac{\partial^{2} u_{i}}{\partial t^{2}}-C_{i j k l} \frac{\partial^{2} u_{k}}{\partial x_{j} \partial x_{l}}=0
$$

where $\rho$ is density, $u_{i}$ is displacement in $x_{i}$ direction, and $C_{i j k l}$ is elastic stiffness tensor. The elastic stiffness tensor $C_{i j k l}$ for a cubic anisotropic system in a coordinate system aligned with crystal's principle axis can be conveniently represented as 6x6 matrix:

$$
C_{n m}=\left(\begin{array}{cccccc}
C_{11} & C_{12} & C_{12} & 0 & 0 & 0 \\
C_{12} & C_{11} & C_{12} & 0 & 0 & 0 \\
C_{12} & C_{12} & C_{11} & 0 & 0 & 0 \\
0 & 0 & 0 & C_{44} & 0 & 0 \\
0 & 0 & 0 & 0 & C_{44} & 0 \\
0 & 0 & 0 & 0 & 0 & C_{44}
\end{array}\right),
$$

where $1 \rightarrow 11,2 \rightarrow 22,3 \rightarrow 33,4 \rightarrow 23,5 \rightarrow 13$, and $6 \rightarrow 12$ convention for relating $n$ and $m$ to $i j$ and $k l$ in in $C_{n m}$ and $C_{i j k l}$, respectively, is implemented. Here we adopted a coordinate system defined with respect to a grain orientation, rather than frame fixed to a laboratory frame. To determine the velocity of ultrasonic wave along a direction given by vector $\left(h_{i}, h_{j}, h_{k}\right)$, we consider a plane wave of the form $u_{k}=U_{k} e^{i \omega\left(h_{j} x_{j} / V-t\right)}$, where $V$ is ultrasonic velocity, $U_{k}$ is 
displacement amplitude of the wave along direction $x_{i}$. Inserting latter into Eq. 2, we arrive at eigenvalue problem:

$$
\left|\begin{array}{ccc}
h_{1}^{2} C_{11}+\left(h_{2}^{2}+h_{3}^{2}\right) C_{44}-\rho V^{2} & h_{1} h_{2}\left(C_{12}+C_{44}\right) & h_{1} h_{3}\left(C_{12}+C_{44}\right) \\
h_{1} h_{2}\left(C_{12}+C_{44}\right) & h_{2}^{2} C_{11}+\left(h_{1}^{2}+h_{3}^{2}\right) C_{44}-\rho V^{2} & h_{2} h_{3}\left(C_{12}+C_{44}\right) \\
h_{1} h_{3}\left(C_{12}+C_{44}\right) & h_{2} h_{3}\left(C_{12}+C_{44}\right) & h_{3}^{2} C_{11}+\left(h_{1}^{2}+h_{2}^{2}\right) C_{44}-\rho V^{2}
\end{array}\right|=0
$$

This eigenvalue problem has three solutions (one quasi-longitudinal and two quasi-shear waves). The analysis outlined above can be conveniently applied to determine the velocity of the ultrasonic wave propagating normal to the surface, assuming that crystallographic orientation of the grain with respect to the surface is available from a different measurement approach. Alternatively one can invert the problem to determine grain's orientation if all 3 different velocities are known. The elastic anisotropy of cubic materials is conveniently represented by the Zener anisotropy ratio $a_{r}=2 C_{44} /\left(C_{11}-C_{12}\right)$. Using literature values for elastic constants $C_{11}=$ $389 \mathrm{MPa}, C_{12}=119 \mathrm{MPa}$ and $C_{44}=59.7 \mathrm{MPa}$, we find $a_{r}=0.44$ in $\mathrm{UO}_{2} .(32)$

\section{Sample Fabrication, Preparation and Characterization}

A sintered polycrystalline depleted $\mathrm{UO}_{2}$ sample was used in this work. The sample was derived from an as-sintered pellet measuring $5.2 \mathrm{~mm}$ in diameter and $4.3 \mathrm{~mm}$ in height, and about $95 \%$ theoretical density. The $\mathrm{O} / \mathrm{U}$ ratio of the pellet was fixed at 2 by cooling the pellet at $\mathrm{Ar}-6 \% \mathrm{H}$ after sintering. The grain size for the samples was increased from $5 \mu \mathrm{m}$ to $50 \mu \mathrm{m}$ by annealing at $2200{ }^{\circ} \mathrm{C}$ under Ar- $6 \% \mathrm{H}$ environment. The final sample was obtained by slicing off a $1 \mathrm{~mm}$ thick segment and polishing one side to a mirror finish. (33)

A dual-beam FEI 3D Quanta focused ion beam (FIB)-scanning electron microscope (SEM) was used to identify and subsequently characterize the grain boundary of interest. First a SEM was used to map the location of the grains on the surface. Then a focused ion beam was used to pattern platinum fiducial marks around the grain boundary of interest. The picosecond 
ultrasonics setup employed in this study uses an optical camera to image the sample surface through the same objective used to focus the pump and probe beams. The fiducial marks are optically visible and are used to locate the boundary of interest. Then Electron Back Scattering Diffraction (EBSD) was used to determine the orientation of grains surrounding the boundary. After the picosecond acoustic measurements were complete, the FIB was used to lift out a lamellae perpendicular to grain boundary line and the surface normal, to reveal the depth resolved cross section of the grain boundary.

\section{Picosecond Ultrasonics Setup}

Our picosecond ultrasonics setup utilizes an ultrafast Ti-sapphire laser oscillator (CoherentMira). The laser system is operated at $800 \mathrm{~nm}$, has a pulse duration of approximately $\sim 1 \mathrm{ps,} \mathrm{a}$ repetition rate of $76 \mathrm{MHz}$ and a pulse energy of $\sim 0.1 \mathrm{~nJ}$. The $400 \mathrm{~nm}$ pump beam is produced by focusing the output of the oscillator onto a beta barium borate (BBO) optical nonlinear crystal. The pump and $800 \mathrm{~nm}$ probe beam are separated using a dichroic mirror placed after the BBO crystal. The pump beam is modulated at $1 \mathrm{MHz}$ using an acousto-optic modulator (Gooch \& Housego). Lockin detection (Stanford Research SRS844) is employed to measure small changes in optical reflectivity of the probe beam associated with the propagating ultrasonic wave. The delay of the pump beam relative to the probe beam is achieved using a mechanical delay line (Newport) placed in the pump leg. Both beams are focused through the same 50x objective and directed at normal incidence angle on the polished surface of the sample. (34) The $\mathrm{UO}_{2}$ sample was mounted on a linear stage. Translation of the linear stage was used to record picosecond acoustic signals at different locations along the surface of the sample.(35)

\section{Results}


An SEM micrograph of the grain boundary region examined using picosecond acoustic is shown in Fig. 2(a). A corresponding EBSD image of the same region is shown in Fig. 2(b). Platinum fiducial marks outlining the approximate position of grain boundary line are clearly visible in the SEM micrograph and were used for positioning the pump and probe beams for optical measurement. The EBSD data confirms the existence of two grains with different orientations. Note that the quality of the image was affected by residue left from patterning the platinum fiducial marks.

Figure 3 shows a typical transient reflectivity signal. The sudden change in reflectivity at $t=0$ is due to ultrafast electron heating followed by a slow decay of the background due to thermal diffusion. High frequency Brillouin oscillations are superimposed on top of this electronic/thermal background. A 3rd order polynomial was used to fit the response (solid line in Fig.3), and subtracted to isolate Brillouin oscillations. Next, we demonstrate how the anisotropic elastic properties manifest themselves when picosecond ultrasonics is applied to a point inside a grain far removed from a boundary. Figure 4 shows Brillouin oscillation observed from two regions (denoted A and B), each corresponding to a different grain. Both sets of Brillouin oscillations can be described by a damped sine wave $\sin (2 \pi f t-\varphi) \exp (-t / \tau)$, where $f$ is the oscillation frequency, $t$ is the pump/probe time delay, $\varphi$ is the phase offset and and $\tau$ is the decay time constant. The best fit to the data (solid lines) is also shown in Fig. 4. Fourier transforms of the corresponding Brillouin oscillations are shown in Fig. 5. The measured frequencies for regions $\mathrm{A}$ and $\mathrm{B}$ are $f_{A}=31.5 \mathrm{GHz}$ (red) and $f_{\mathrm{B}}=30.5 \mathrm{GHz}$ (blue). Using Eq. 1 and $n=2.28$ for $\mathrm{UO}_{2}$ at $800 \mathrm{~nm}(36)$ we calculate $v_{A}=5,530 \mathrm{~m} / \mathrm{s}$ and $v_{B}=5,350 \mathrm{~m} / \mathrm{s}$. Similar intra-grain results have been recently reported for a polycrystalline $\mathrm{BiFeO}_{3}$ compound.(35) 
The bulk wave velocities along a direction normal to the surface are obtained using the Christoffel equation (Eq. 4). Using the grain orientation $\left(h_{i}, h_{j}, h_{k}\right)$ obtained from the EBSD data and literature values for the elastic constants, we calculate the longitudinal wave velocities to be $v_{A}=5,510 \mathrm{~m} / \mathrm{s}$ and $v_{B}=5,380$. These values agree well with the values obtained from the analysis of the Brillouin oscillations.

Close examination of Fourier transform shown in Fig. 5 reveals an additional peak at $f_{\mathrm{A}}{ }^{\prime}=17.2$ $\mathrm{GHz}$ in the spectra associated with region A corresponding to $v_{\mathrm{A}}{ }^{\prime}=3020 \mathrm{~m} / \mathrm{s}$. This corresponds closely to the calculated fast quasi-shear wave velocity of $3000 \mathrm{~m} / \mathrm{s}$. The quasi shear wave is composed of shear strain and longitudinal strain components. Detection of the quasi shear mode with normal incidence light is due to first-order photoelastic coupling with the longitudinal component of the shear wave. The ability to detect longitudinal and shear waves calls for further analysis to determine if this approach can be used to determine the crystal orientation of the grain.

In the next step, we interrogate the region between the two grains, i.e. near the grain boundary. Figure 6 shows the Brillouin oscillations recorded at several different spots along the line that intersects the boundary line at $90^{\circ}$ (see Fig. 2a). Sufficiently away from the interface on both sides of the interface at positions $s_{1}=5 \mu \mathrm{m}$ and $s_{6}=14 \mu \mathrm{m}$, the oscillations can be described by a single frequency. The situation is different for positions in the close vicinity of the boundary as can be seen in Fig. 7. The oscillation recorded at position $s_{3}=10 \mu \mathrm{m}$ cannot be fitted with a single frequency sine wave (red line becoming dashed). A best fit is obtained when the waveform is divided in two sections. The first is represented by oscillations at frequency $f_{\mathrm{A}}$ (red line) and the second at $f_{\mathrm{B}}$ (blue line), corresponding to grains $\mathrm{A}$ and $\mathrm{B}$ respectively. The transition time is used as the only fitting parameter. At $s_{3}$, the transition from A to B takes place at $t_{3}=655$ 
ps. The step change in oscillation amplitude upon passing into the second grain is due to loss of energy caused by acoustic reflection at the interface.

The orientation of the subsurface boundary relative to the free surface can be estimated if we assume that our probe spot size is infinitesimally small. Using the measured ultrasonic longitudinal velocity, the depth below the surface at which the ultrasonic wave propagates across the grain boundary is calculated to be $d_{3}=v_{\mathrm{A}} \times t_{3}=3.63 \mu \mathrm{m}$. Best fit data is compared with several sets of experimental Brillouin oscillations in Fig. 6. Red lines represent a section of oscillations corresponding to grain A and blue lines correspond to grain B. The result of this analysis is used to reconstruct the subsurface grain boundary in the right pane of Fig. 6. For $s \leq s_{1}$, we only observe oscillations corresponding to grain A across the whole depth. In the other limit $s \geq s_{5}$, only grain B is detected. In the intermediate range between $s_{2} \leq s \leq s_{4}$, both grains $\mathrm{A}$ and B are observed. Two depth values closest to the free surface at $s_{3}$ and $s_{4}$ are used to determine the tilt of the grain boundary relative to the surface normal to be $\theta=29^{\circ}$.

To verify our observations we performed cross sectional analysis of the grain boundary using Transmission EBSD. A FIB was used to lift out a lamellae perpendicular to the grain boundary line on the surface, corresponding to the cross section of the region studied. Figure 8a shows the trench resulting from the lift-out. A projection corrected version of the data in Fig. 8(a), shown in Fig.8(b), is used to determine the grain boundary tilt relative to the surface normal. The value measured using the FIB liftout, $\theta=24^{\circ}$, is in reasonable agreement with our Brillouin oscillation measurements. The EBDS image of the FIB lamella shown in Fig. 8(c) confirms the existence of two grains with distinct orientation.

The lift outs were limited to the depth of $2.7 \mu \mathrm{m}$, which can be considered a practical limit for FIB lift-out approach. It is important to note that the picosecond ultrasonic approach can image 
grain structure below this limit nondestructively. In fact the ultrasonic data at depth of $5.2 \mu \mathrm{m}$ indicates that the grain boundary begins to curve towards the plane of the free surface, which would explain a discrepancy between angles obtained from picosecond acoustics and FIB lift-out analysis.

\section{Conclusions}

The results presented above demonstrate the capability of picosecond ultrasonics to nondestructively provide volumetric information regarding grain boundary structure in polycrystalline $\mathrm{UO}_{2}$. By tuning the wavelength of the pump and probe this approach could be extended to a larger group of semiconducting materials. Also by coating a thin metallic film on the surface this approach could be extended to transparent materials. While current implementation involved a single line scan, future work involving raster scanning can be used to more completely define subsurface grain boundary structure. A more comprehensive analysis, including the consequences of acoustic diffraction, the finite size of the probe beam and spreading of the probe beam with depth, must be considered before we can correctly define resolution limits. However, using our simplified analysis, we estimate the lateral resolution to be on the order of $500 \mathrm{~nm}$ and the maximum probe depth to be on the order of $10 \mu \mathrm{m}$. While these values are at least on order of magnitude larger than that of SEM based EBSD method, this approach does not require serial sectioning to see below the surface of the material and offers to provide volumetric information over larger area than accessible using FIB liftout for depth resolved analysis.

Capabilities of the picosecond acoustic can be further expanded by taking into consideration recent reports where the structure of interface resulted in additional modifications to the Brillouin oscillations, $(31,37)$ such as modulation in amplitude and emergence of a phase lag 
upon propagation through a distinct layer. We speculate that these effects can be leveraged to study impurity segregation at grain boundaries using picosecond ultrasonics.

\section{Acknowledgments}

This material is based upon work supported as part of the Center for Materials Science of Nuclear Fuel, an Energy Frontier Research Center funded by the U.S. Department of Energy, Office of Science, Office of Basic Energy Sciences under Award Number FWP 1356. The authors would also like to acknowledge the use of the FIB and EBSD instrumentation at the Center for Advanced Energy Studies which is supported by the U.S. Department of Energy, Office of Nuclear Energy under DOE Idaho Operations Office Contract DE-AC07-051D14517. 
Figure 1. Schematics of experimental approach used for observation of Brillouin oscillations. Ultrasonic wave excited by a thermoelastic deformation caused by the pump beam and propagating normal to the surface with velocity $v_{\mathrm{s}}$ is represented by a dashed line. Two reflections of the probe beam one from the surface (solid vertical line) and one from the front of ultrasonic wave (dashed vertical line) result in an interference pattern whose period and frequency is proportional to ultrasonic velocity and index of refraction of probe beam in the material. This interference pattern is observed in time resolved reflectivity change of the probe beam. In the actual experiment, the probe is at normal incidence to the surface, here it is shown at angle to emphasize two reflections. Cartesian coordinate system used to calculate the velocity based on ultrasonic wave equation is defined with respect to the grain's crystallographic orientation.

Figure 2. (a) An SEM image of an area around two neighboring grains after the platinum fiducial marks were deposited. Dotted line corresponds to a location of grain boundary line. Dashed thick line indicates the location of the line scan along which Brillouin oscillations were recorded and the lift-out was made for cross-sectional characterization. (b) EBSD image of the same area revealing two distinct grains. The results obtained from region above the platinum marks are noisy and have no physical meaning. Crystallographic orientation revealed by EBSD for grain A is (9 10 24) [26 3 -11] and B is (1 3 4) [20 - 16 7]. Orientation of the grains with respect to the surface is depicted by rotated cubes.

Figure 3. Time resolved change in the reflectivity of $800 \mathrm{~nm}$ probe in $\mathrm{UO}_{2}$ following an excitation by $400 \mathrm{~nm}$ pump pulse. Brillouin oscillations are superimposed over the electronic or thermal background response. Solid line is a polynomial fit used to subtract the background to isolate Brillouin oscillations.

Figure 4. Brillouin oscillations from two regions of the polycrystalline $\mathrm{UO}_{2}$ sample, corresponding to grains A (red diamonds) and B (blue circles). Solid lines are fits based on damped sine waves. Both have noticeably different periods of oscillations.

Figure 5. Fourier transforms of Brillouin oscillations corresponding to grain A (red line) and grain B (blue line). Primary peaks at $31.5 \mathrm{GHz}$ and $30.5 \mathrm{GHz}$ correspond to longitudinal waves propagating normal to the surface along grain A and B respectively. Additional peak $17.2 \mathrm{GHz}$ corresponds to one of the quasi-shear waves in grain $\mathrm{A}$. 
Figure 6. Mapping of subsurface grain boundary orientation using picosecond ultrasonics. Left: Brillouin oscillations in the vicinity of the grain boundary. Each transient corresponds to a point along a line drawn normal to the boundary line (Fig. 3a). Red and blue solid lines correspond to sections of the experimental data that are best fitted with oscillations at frequency corresponding to grains $\mathrm{A}$ and $\mathrm{B}$, respectively. Right: An image produced using the analysis of Brillouin oscillations. The ultrasonic data at depth of $5.2 \mu \mathrm{m}$ indicates that the grain boundary begins to curve towards the plane of the free surface.

Figure 7. Brillouin oscillations observed at a point close to the interface. Frequency of the oscillations changes at $t=650 \mathrm{ps}$, corresponding to the change in the velocity of ultrasonic wave as it initially propagates along grain A and then continues to grain B.

Figure 8. Destructive measurement of subsurface grain boundary orientation using electron microscopy: (a) Ion beam-induced secondary electron image of the grain boundary region, previously ion milled orthogonal to both the surface and boundary line. (b) Measurement of the boundary angle after projection-correction of (a). (c) Transmission EBSD of the local area, confirming grain boundary location and crystallographic orientation of grains. 


\section{References:}

1. A. P. Sutton, R. W. Balluffi, Interfaces in crystalline materials. Monographs on the physics and chemistry of materials (Clarendon Press ; Oxford University Press, Oxford New York, 1995).

2. M. Mogensen, N. M. Sammes, G. A. Tompsett, Physical, chemical and electrochemical properties of pure and doped ceria. Solid State Ionics 129, 63-94 (2000).

3. Z. J. Wang, J. E. Alaniz, W. Y. Jang, J. E. Garay, C. Dames, Thermal Conductivity of Nanocrystalline Silicon: Importance of Grain Size and Frequency-Dependent Mean Free Paths. Nano Letters 11, 2206-2213 (2011).

4. C. Ronchi, M. Sheindlin, D. Staicu, M. Kinoshita, Effect of burn-up on the thermal conductivity of uranium dioxide up to 100.000 MWdt(-1). Journal of Nuclear Materials 327, 58-76 (2004).

5. M. Khafizov et al., Thermal Conductivity in Nanocrystalline Ceria Thin Films. Journal of the American Ceramic Society 97, 562-569 (2014).

6. D. G. Cahill et al., Nanoscale thermal transport. Journal of Applied Physics 93, 793-818 (2003).

7. D. A. Andersson et al., Multiscale simulation of xenon diffusion and grain boundary segregation in UO2. Journal of Nuclear Materials 462, 15-25 (2015).

8. D. Shrader et al., Ag diffusion in cubic silicon carbide. Journal of Nuclear Materials 408, 257-271 (2011).

9. P. Heitjans, S. Indris, Diffusion and ionic conduction in nanocrystalline ceramics. Journal of PhysicsCondensed Matter 15, R1257-R1289 (2003).

10. M. A. Meyers, A. Mishra, D. J. Benson, Mechanical properties of nanocrystalline materials. Progress in Materials Science 51, 427-556 (2006).

11. M. L. Taheri, J. T. Sebastian, B. W. Reed, D. N. Seidman, A. D. Rollett, Site-specific atomic scale analysis of solute segregation to a coincidence site lattice grain boundary. Ultramicroscopy 110, 278-284 (2010).

12. M. Hong et al., The role of charge and ionic radius on fission product segregation to a model UO2 grain boundary. Journal of Applied Physics 113, (2013).

13. X.-M. Bai, Y. Zhang, M. R. Tonks, Testing thermal gradient driving force for grain boundary migration using molecular dynamics simulations. Acta Materialia 85, 95-106 (2015).

14. F. Dherbey, F. Louchet, A. Mocellin, S. Leclercq, Elevated temperature creep of polycrystalline uranium dioxide: from microscopic mechanisms to macroscopic behaviour. Acta Materialia 50, 1495-1505 (2002).

15. I. Szlufarska, A. Nakano, P. Vashishta, A crossover in the mechanical response of nanocrystalline ceramics. Science 309, 911-914 (2005).

16. I. M. Robertson et al., Towards an integrated materials characterization toolbox. Journal of Materials Research 26, 1341-1383 (2011).

17. Y. Huang, F. J. Humphreys, Measurements of grain boundary mobility during recrystallization of a single-phase aluminium alloy. Acta Materialia 47, 2259-2268 (1999).

18. T. Gorkaya, K. D. Molodov, D. A. Molodov, G. Gottstein, Concurrent grain boundary motion and grain rotation under an applied stress. Acta Materialia 59, 5674-5680 (2011).

19. G. E. Ice, B. C. Larson, 3D X-ray crystal microscope. Advanced Engineering Materials 2, 643-646 (2000).

20. S. Schmidt et al., Watching the growth of bulk grains during recrystallization of deformed metals. Science $\mathbf{3 0 5}$, 229-232 (2004).

21. D. Gostovic, J. R. Smith, D. P. Kundinger, K. S. Jones, E. D. Wachsman, Three-dimensional reconstruction of porous LSCF cathodes. Electrochemical and Solid State Letters 10, B214-B217 (2007).

22. M. D. Uchic, L. Holzer, B. J. Inkson, E. L. Principe, P. Munroe, Three-dimensional microstructural characterization using focused ion beam tomography. Mrs Bulletin 32, 408-416 (2007). 
23. M. Teague, B. Gorman, Utilization of dual-column focused ion beam and scanning electron microscope for three dimensional characterization of high burn-up mixed oxide fuel. Progress in Nuclear Energy 72, 67-71 (2014).

24. C. Sorensen, J. A. Basinger, M. M. Nowell, D. T. Fullwood, Five-Parameter Grain Boundary Inclination Recovery with EBSD and Interaction Volume Models. Metallurgical and Materials Transactions a-Physical Metallurgy and Materials Science 45A, 4165-4172 (2014).

25. C. Mechri et al., Depth-profiling of elastic inhomogeneities in transparent nanoporous low-k materials by picosecond ultrasonic interferometry. Applied Physics Letters 95, (2009).

26. V. Gusev, A. M. Lomonosov, P. Ruello, A. Ayouch, G. Vaudel, Depth-profiling of elastic and optical inhomogeneities in transparent materials by picosecond ultrasonic interferometry: Theory. Journal of Applied Physics 110, (2011).

27. T. T. Meek, B. von Roedern, P. G. Clem, R. J. Hanrahan, Some optical properties of intrinsic and doped UO2 thin films. Materials Letters 59, 1085-1088 (2005).

28. Y. Q. An et al., Ultrafast Hopping Dynamics of 5 f Electrons in the Mott Insulator UO2 Studied by Femtosecond Pump-Probe Spectroscopy. Physical Review Letters 106, 4 (2011).

29. O. B. Wright, B. Perrin, O. Matsuda, V. E. Gusev, Ultrafast carrier diffusion in gallium arsenide probed with picosecond acoustic pulses. Physical Review B 64, (2001).

30. C. Thomsen, H. T. Grahn, H. J. Maris, J. Tauc, SURFACE GENERATION AND DETECTION OF PHONONS BY PICOSECOND LIGHT-PULSES. Physical Review B 34, 4129-4138 (1986).

31. A. Steigerwald et al., Semiconductor point defect concentration profiles measured using coherent acoustic phonon waves. Applied Physics Letters 94, (2009).

32. I. J. Fritz, ELASTIC PROPERTIES OF UO2 AT HIGH-PRESSURE. Journal of Applied Physics 47, 43534358 (1976).

33. B. Valderrama et al., Effect of Grain Boundaries on Krypton Segregation Behavior in Irradiated Uranium Dioxide. Jom 66, 2562-2568 (2014).

34. M. Khafizov, D. H. Hurley, Measurement of thermal transport using time-resolved thermal wave microscopy. Journal of Applied Physics 110, 083525 (2011).

35. M. Lejman et al., Giant ultrafast photo-induced shear strain in ferroelectric BiFeO3. Nature Communications $\mathbf{5}$, (2014).

36. J. Schoenes, OPTICAL-PROPERTIES AND ELECTRONIC-STRUCTURE OF UO2. Journal of Applied Physics 49, 1463-1465 (1978).

37. D. Yarotski et al., Characterization of irradiation damage distribution near TiO2/SrTiO3 interfaces using coherent acoustic phonon interferometry. Applied Physics Letters 100, (2012). 


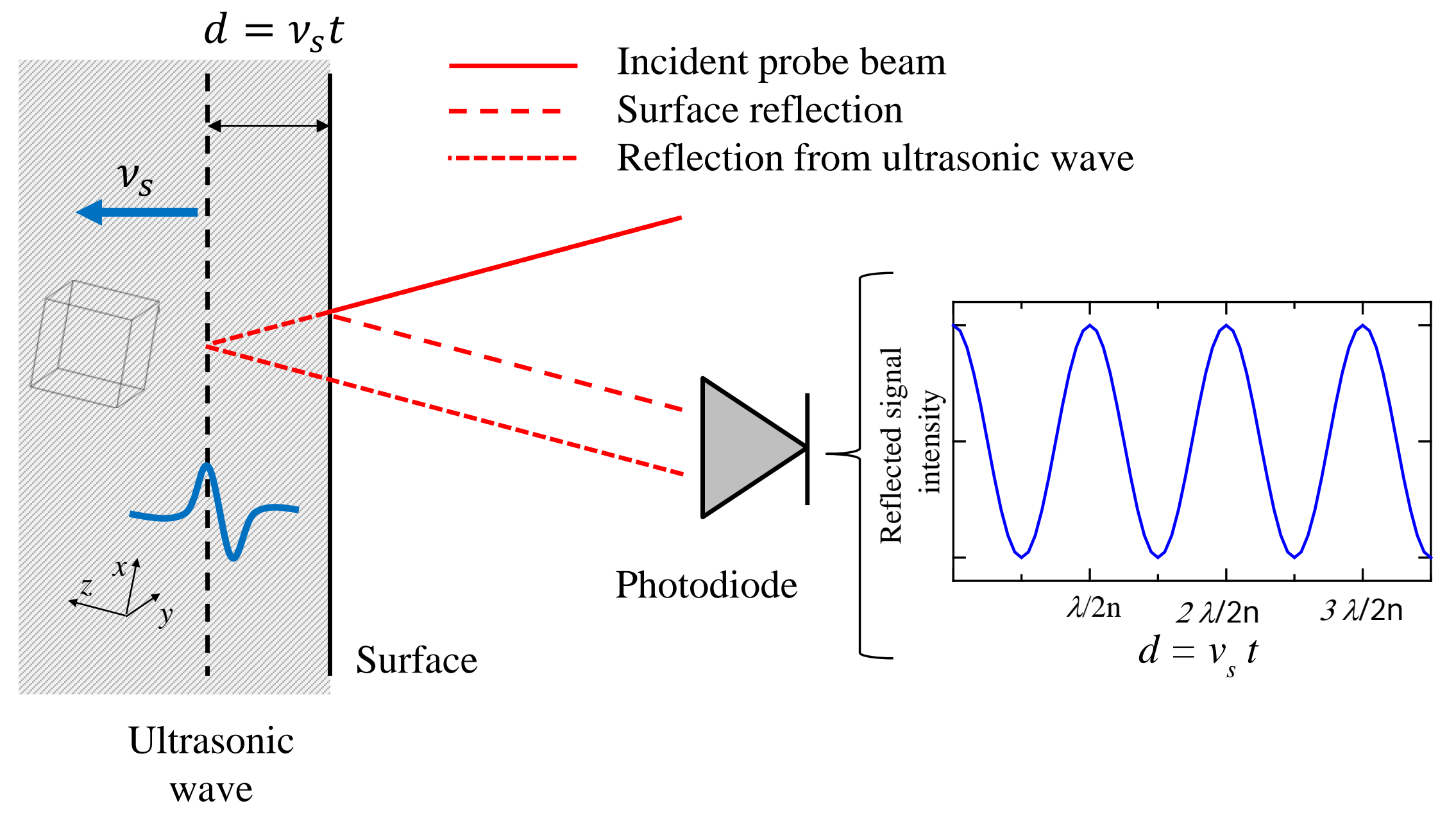


(a)

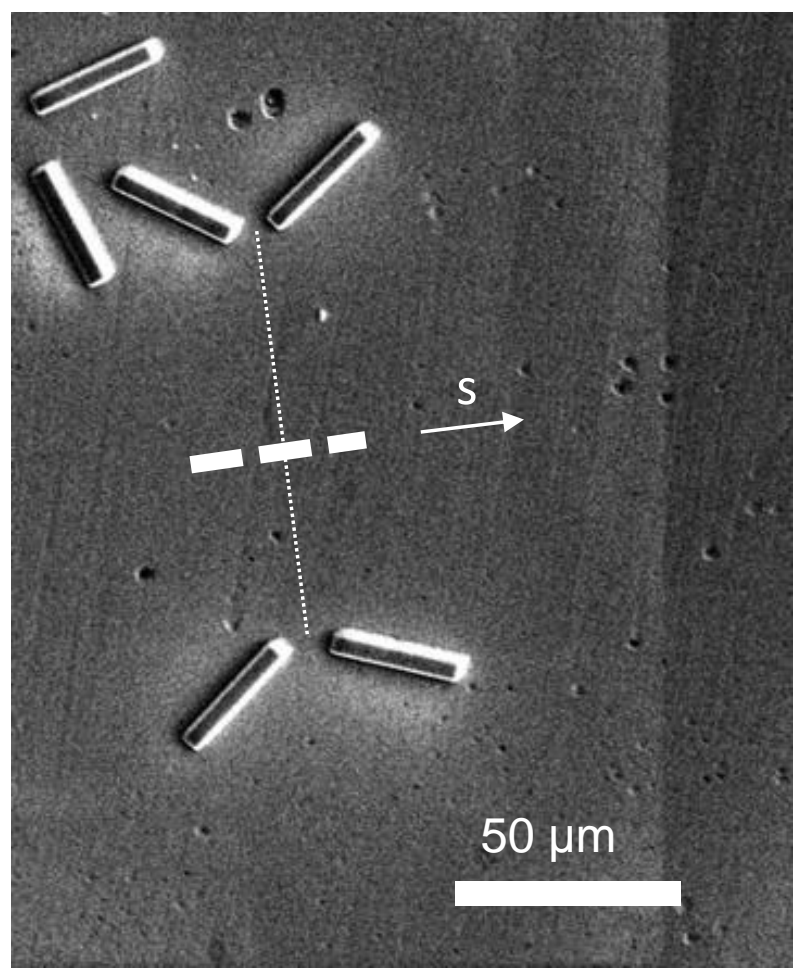

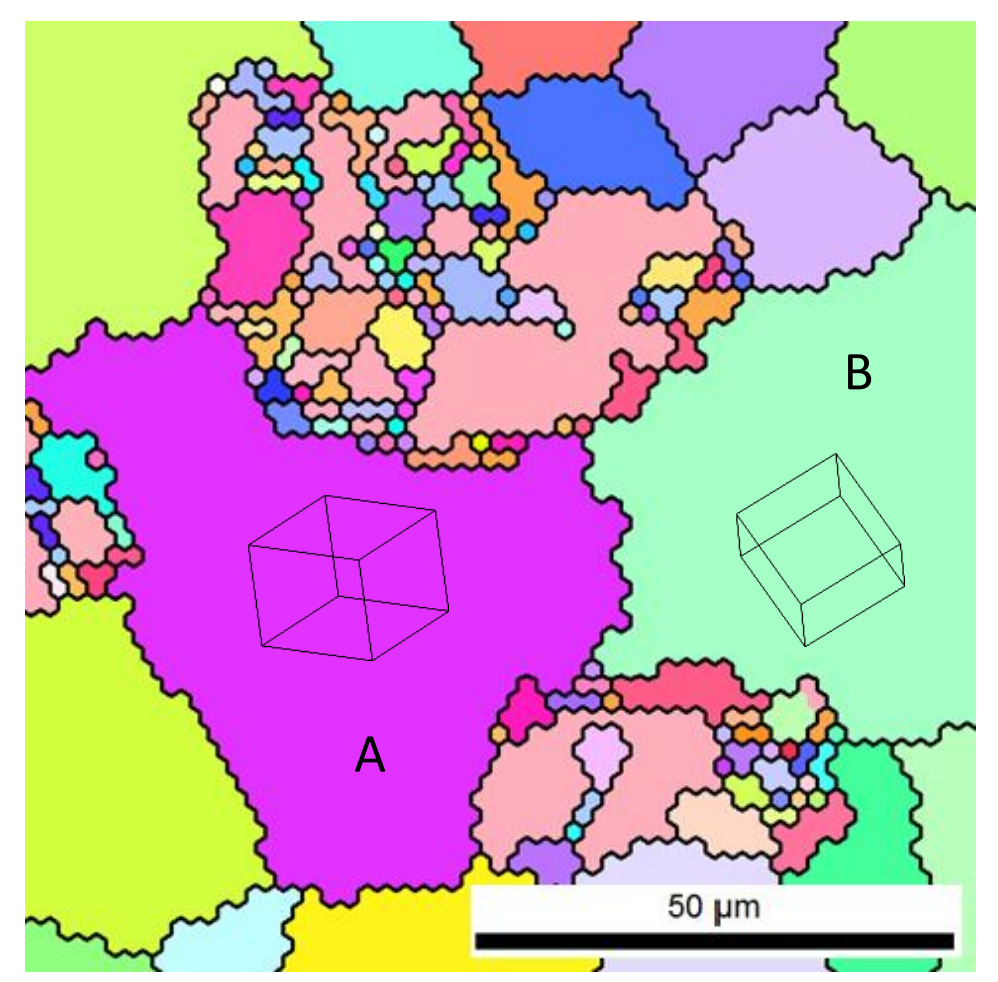

(b) 


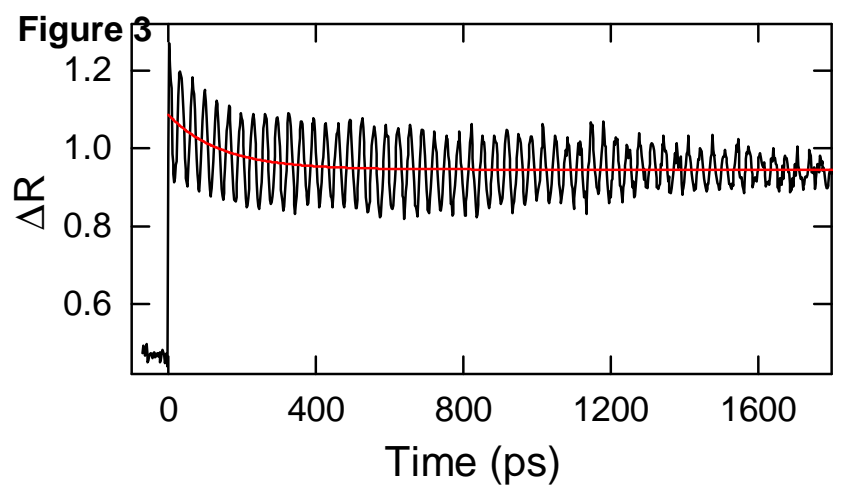


Figure 5

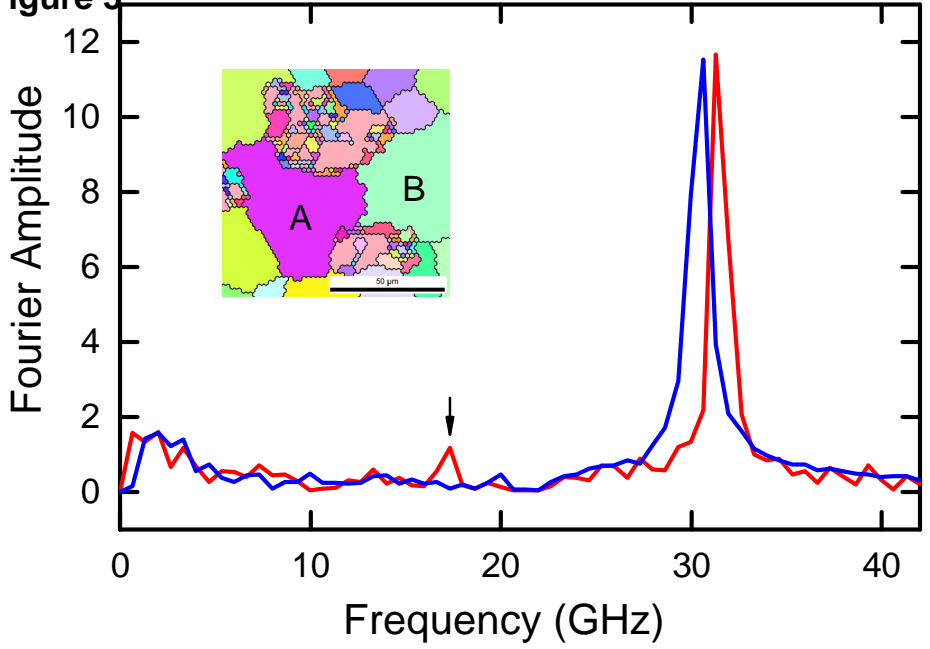




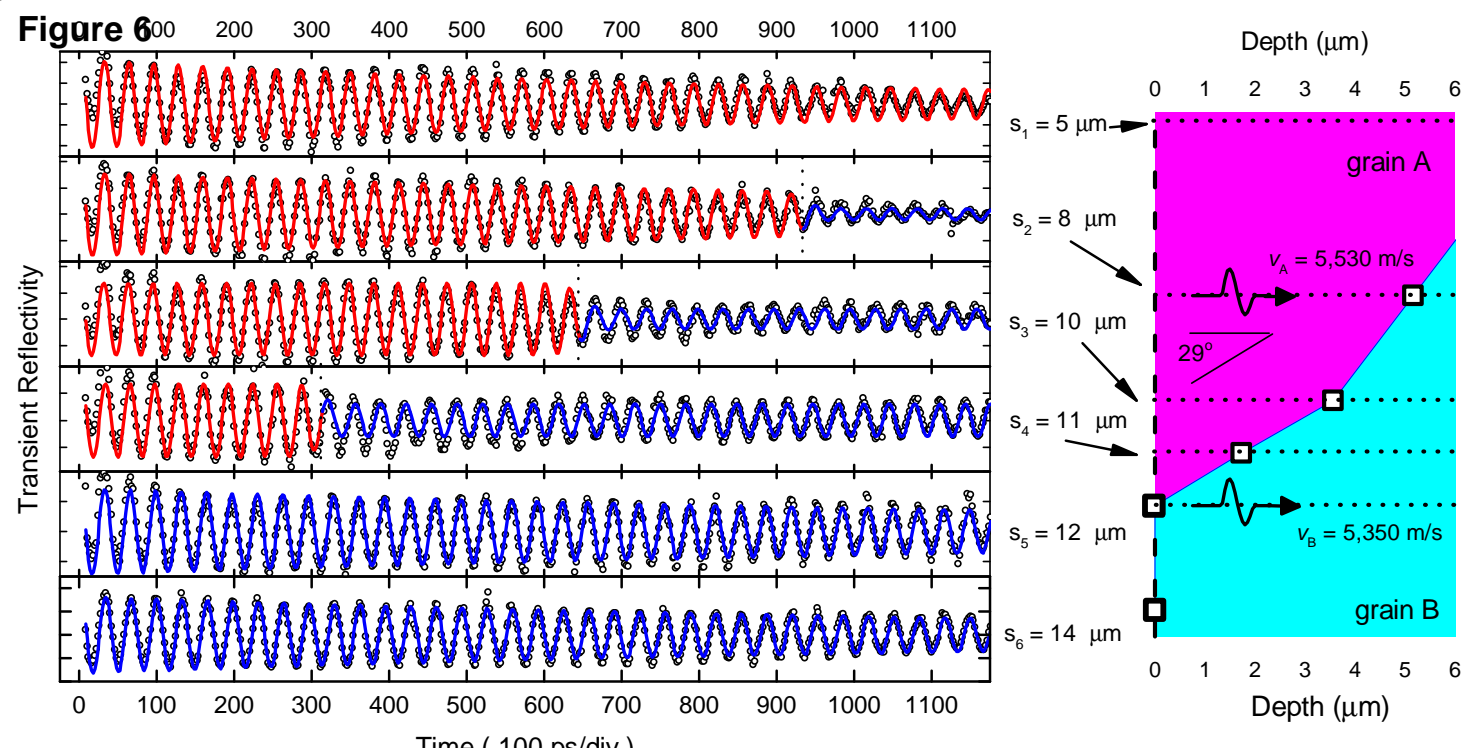


Figure 7

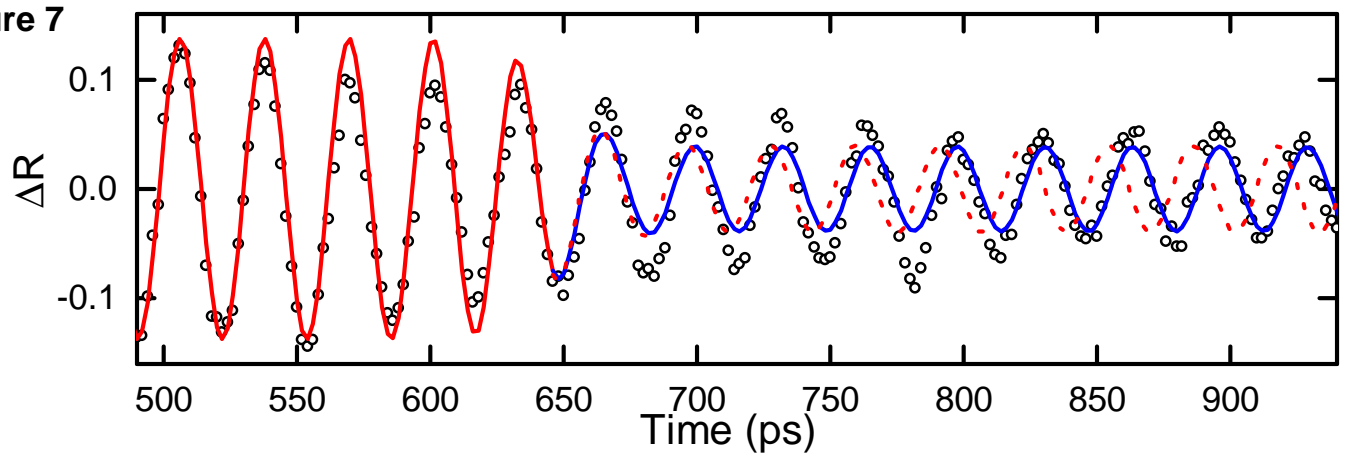



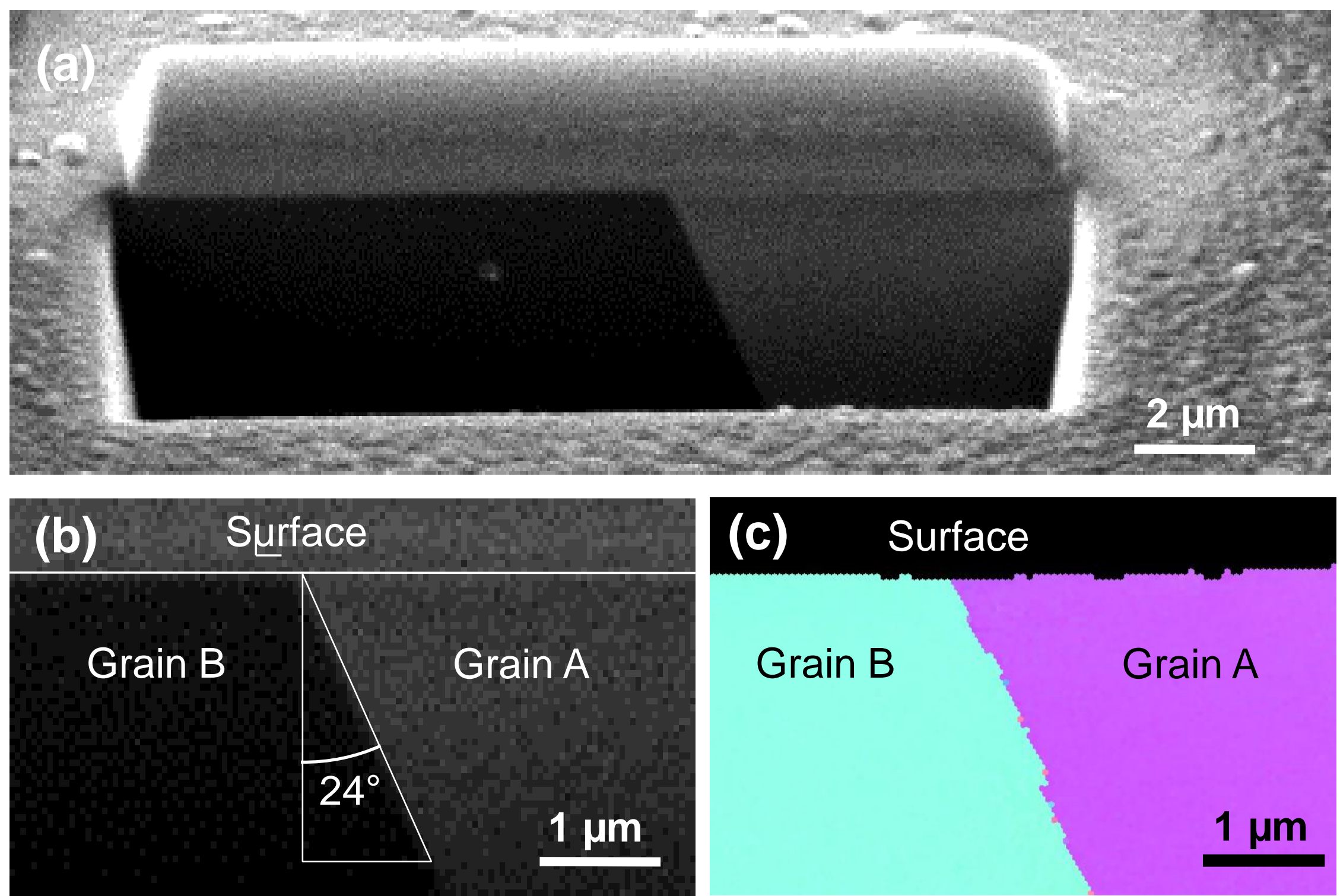

(c) Surface

Grain B

Grain A 


\section{*Graphical Abstract}

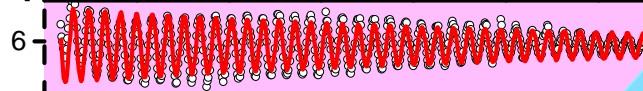

$7+$

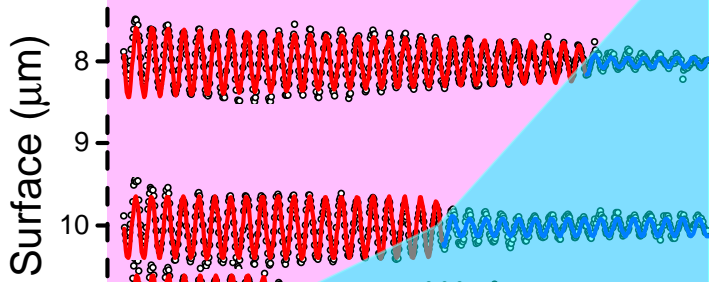
11 th

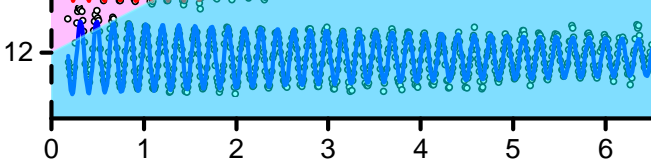
Depth $(\mu \mathrm{m})$ 